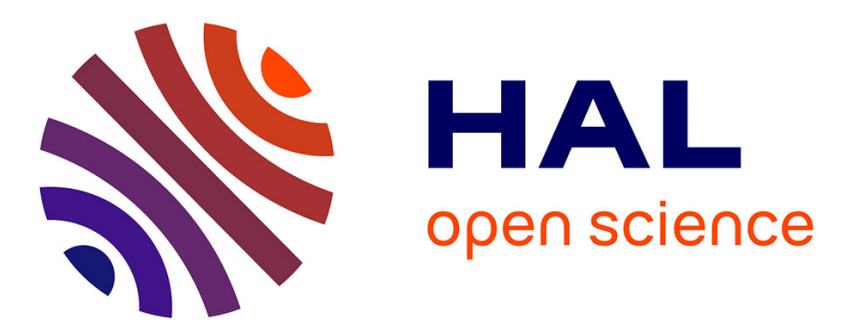

\title{
THz Emissions from Air-Plasmas Created by Mid-and Far-Infrared Two-Color Femtosecond Pulses
}

A. Nguyen, L. Bergé, P. González de Alaiza Martínez, I. Thiele, Stefan Skupin

\section{To cite this version:}

A. Nguyen, L. Bergé, P. González de Alaiza Martínez, I. Thiele, Stefan Skupin. THz Emissions from Air-Plasmas Created by Mid-and Far-Infrared Two-Color Femtosecond Pulses. CLEO: Applications and Technology, 2018, San Jose, United States. 10.1364/CLEO_AT.2018.JTu2A.121 . hal-01795124

\section{HAL Id: hal-01795124 \\ https://hal.science/hal-01795124}

Submitted on 18 May 2018

HAL is a multi-disciplinary open access archive for the deposit and dissemination of scientific research documents, whether they are published or not. The documents may come from teaching and research institutions in France or abroad, or from public or private research centers.
L'archive ouverte pluridisciplinaire HAL, est destinée au dépôt et à la diffusion de documents scientifiques de niveau recherche, publiés ou non, émanant des établissements d'enseignement et de recherche français ou étrangers, des laboratoires publics ou privés. 


\title{
THz Emissions from Air-Plasmas Created by Mid- and Far-Infrared Two-Color Femtosecond Pulses
}

\author{
A. Nguyen ${ }^{1}$, L. Bergé ${ }^{1}$, P. González de Alaiza Martínez ${ }^{2}$, I. Thiele ${ }^{2}$, and S. Skupin ${ }^{3}$ \\ ${ }^{1}$ CEA, DAM, DIF, F-91297 Arpajon, France \\ ${ }^{2}$ Univ. Bordeaux - CNRS - CEA, Centre Lasers Intenses et Applications, UMR 5107, 33405 Talence, France \\ ${ }^{3}$ Institut Lumière Matière, UMR 5306 Université Lyon 1 - CNRS, Université de Lyon, 69622 Villeurbanne, France \\ alisee.nguyen@cea.fr
}

\begin{abstract}
We study THz emission by two-color femtosecond filaments in air using mid- to far-infrared pump wavelengths. 3D numerical simulations show that $10.6-\mu \mathrm{m}$ laser pulses can produce $\mathrm{THz}$ fields with unequaled $\mathrm{mJ}$ energies and $\mathrm{GV} / \mathrm{m}$ amplitudes.
\end{abstract}

OCIS codes: $190.7110,260.3090,020.2649$

\section{Introduction}

Terahertz $(\mathrm{THz})$ science is attracting a broad interest because many molecular fingerprints belong to the THz range [1]. A well-known technique to create efficient $\mathrm{THz}$ emitters is to focus a two-color femtosecond light pulse into air, creating a plasma that acts as frequency converter [2]. Recent studies [3,4] showed that increasing the pump wavelength can enhance the laser-to- $\mathrm{THz}$ conversion efficiency which is usually limited to about $10^{-4}$ for a $800 \mathrm{~nm}$ pump pulse. Moreover, it is known that the pump power contained in a filament is limited to a few times the critical power for selffocusing $P_{\mathrm{cr}}$ before undesired spatial breakup and multiple filamentation kicks in [5]. Because $P_{\mathrm{cr}}$ scales quadratically with the pump wavelength, increasing the latter makes filaments with much higher energies possible. Longer laser wavelengths, e.g., $3.9 \mu \mathrm{m}$, are nowadays accessible in the femtosecond regime and energetic, 100 -fs $\mathrm{CO}_{2}$ laser pulses operating at $10.6 \mu \mathrm{m}$ should be available in the coming years [6]. We here focus on intense, broadband $\mathrm{THz}$ fields produced by such long-wavelength pulses. By means of a three-dimensional (3D) unidirectional solver [4], we study $\mathrm{THz}$ generation by two-color femtosecond pulses ionizing air. Our numerical results evidence unprecedented $\mathrm{THz}$ energy yields and conversion efficiencies achieved by 10.6- $\mu \mathrm{m}$ pump pulses along extended propagation distances.

\section{THz spectra and supercontinuum generation of mid- and far-infrared pulses}

In our simulations, the input light field has a Gaussian profile in space and time with $2.5-\mathrm{mm} 1 / e^{2}$ transverse radius and 100-fs FWHM duration. It is composed of the fundamental (FH) pulse operating at the carrier wavelength $\lambda_{0}$ and $10 \%$ of second harmonic ( $\mathrm{SH}$ ) in energy. The overall beam is linearly focused by a converging lens of $30-\mathrm{cm}$ focal length. All pulses have an input power equal to $1.73 P_{\mathrm{cr}}$ of the $\mathrm{FH}$ pulse and the dispersion map includes HITRAN data for $\mathrm{CO}_{2}$ and water absorption [8]. Peak intensities of $\sim 200 \mathrm{TW} / \mathrm{cm}^{2}$ produce electron densities above $10^{17} \mathrm{~cm}^{-3}$. When increasing the pump wavelength and pump energy $\left(P_{\mathrm{cr}} \propto \lambda_{0}^{2}\right)$, longer filaments are promoted and they start to self-focus at shorter propagation distances. Figure 1(a) illustrates the resulting THz energy yields below $10 \mathrm{THz}$ that reach the $0.1 \mathrm{~mJ}$ level for $3.9-\mu \mathrm{m}$ pumps and several $\mathrm{mJ}$ for $10.6-\mu \mathrm{m}$ lasers. Figures $1(\mathrm{~b}, \mathrm{c}, \mathrm{d})$ detail the $\mathrm{THz}$ spectra computed at the distance of maximum $\mathrm{THz}$ field production, i.e., where the on-axis $\mathrm{THz}$ fields attain their maxima. Because an important question has been the role of Kerr-induced four-wave mixing compared to that of photocurrents [7], Figs. 1(b,c,d) also compare the spectra computed with or without the plasma terms before the linear focus. The presence of plasma clearly increases the $\mathrm{THz}$ spectral intensity by at least three orders of magnitude and shifts the frequency centroid down to $0.2 \mathrm{THz}$ for the three pump wavelengths. Hence, plasma generation - even when it involves low electron densities $\sim 10^{15-17} \mathrm{~cm}^{-3}$ - is here the key player in THz pulse generation.

Figures 1(e,g,i) display the entire optical spectrum developed for the three FH wavelengths along the propagation axis. When increasing the pump wavelength, high-order harmonics are produced more efficiently and form a broader supercontinuum through the plasma nonlinearity and by self- and cross-phase modulation. They are able to contribute to photoionization. For a near-IR pump $(0.8 \mu \mathrm{m})$, the distance of maximum $\mathrm{THz}$ generation coincides with that of maximum plasma generation close to focus. For larger wavelengths, maximum THz generation shifts towards shorter propagation distances. For a pump wavelength of $10.6 \mu \mathrm{m}$, THz production is maximum at $z \approx 20 \mathrm{~cm}$ only, well before 

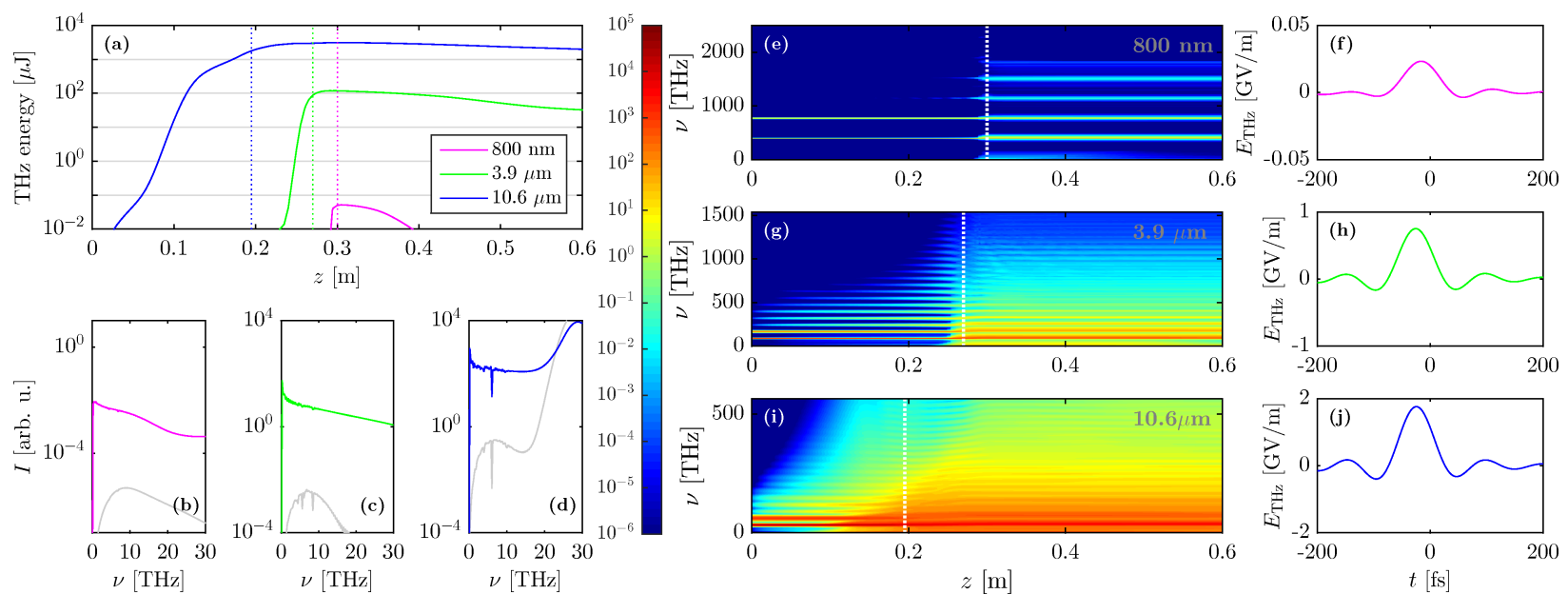

Fig. 1. (a) THz energies numerically computed for two-color Gaussian pulses with FH wavelengths $\lambda_{0}=0.8,3.9$ and $10.6 \mu \mathrm{m}$. Vertical dotted lines indicate the distances of maximum $\mathrm{THz}$ production. (b,c,d) THz spectra at these distances, namely, (b) $z=30 \mathrm{~cm}$, (c) $z=27 \mathrm{~cm}$ and (d) $z=19.5 \mathrm{~cm}$. Gray curves correspond to the same plotted quantities without plasma. Note the absorption line $\sim 6 \mathrm{THz}$ due to water vapor. (e,g,i) Intensity spectra along the propagation axis. (f,h,j) On-axis THz fields obtained by filtering (20th-order Butterworth) the total field in the frequency range $v<10 \mathrm{THz}$.

maximum plasma generation sets in, which is mainly due to high-order harmonics that do no longer contribute to $\mathrm{THz}$ generation. Figures $1(\mathrm{f}, \mathrm{h}, \mathrm{j})$ reveal that on-axis $\mathrm{THz}$ fields induced by a $10.6-\mu \mathrm{m}$ pump are two orders of magnitude higher than with a near-IR pump pulse, and twice as high as with a 3.9- $\mu \mathrm{m}$ pump pulse. Moreover, the transverse $\mathrm{THz}$ field profile broadens with the pump wavelength (not shown). The THz conversion efficiency obtained at $10.6 \mu \mathrm{m}$ is thus three times the one at $3.9 \mu \mathrm{m}$, that is $3 \%$ versus $1 \%$, respectively. In addition, we report much smaller angles of the conical THz emission and a better coupling between the two colors along extended propagation ranges, which renders filamentation setups using $\mathrm{CO}_{2}$ lasers particularly promising for further applications in $\mathrm{THz}$ spectroscopy.

\section{Conclusion}

In summary, we numerically showed that two-color ultrashort $\mathrm{CO}_{2}$ laser filaments can provide $\mathrm{THz}$ conversion efficiencies up to 3\%. Because far-infrared femtosecond filaments contain hundreds of $\mathrm{mJ}$ and are able to self-channel over long distances along which $\mathrm{THz}$ production is stable, they can supply $\mathrm{THz}$ energy yields of several $\mathrm{mJ}$. Such energy levels are record values for $100-\mathrm{mJ}$ pump pulses operating at moderately high (non-relativistic) laser intensities.

\section{References}

1. M. Tonouchi, "Cutting-edge terahertz technology," Nature Photon. 1, 97 (2007)

2. K. Y. Kim, A. J. Taylor, J. H. Glownia and G. Rodriguez, "Coherent control of terahertz supercontinuum generation in ultrafast laser-gas interactions," Nature Photon. 2, 605 (2008)

3. M. Clerici et al., "Wavelength scaling of terathertz generation by gas ionization," Phys. Rev. Phys. 110, 253901 (2013)

4. A. Nguyen et al., "Spectral dynamics of $\mathrm{THz}$ pulses generated by two-color laser filaments in air: the role of Kerr nonlinearities and pump wavelength," Opt. Express 25, 4720 (2017)

5. A.J. Campillo, S.L. Shapiro, B.R. Suydam, "Periodic breakup of optical beams due to selffocusing," Appl. Phys. Lett. 23, 628 (1973)

6. I. V. Pogorelsky et al., "BESTIA - The next generation ultra-fast $\mathrm{CO}_{2}$ laser for advanced accelerator research," Nucl. Inst. Meth. Phys. Res. A 829, 432 (2016)

7. V. A. Andreeva et al., "Ultrabroad terahertz spectrum generation from an air-based filament plasma," Phys. Rev. Lett. 116, 063902 (2016)

8. Hitran on the web (2017), http://hitran.iao.ru/. 